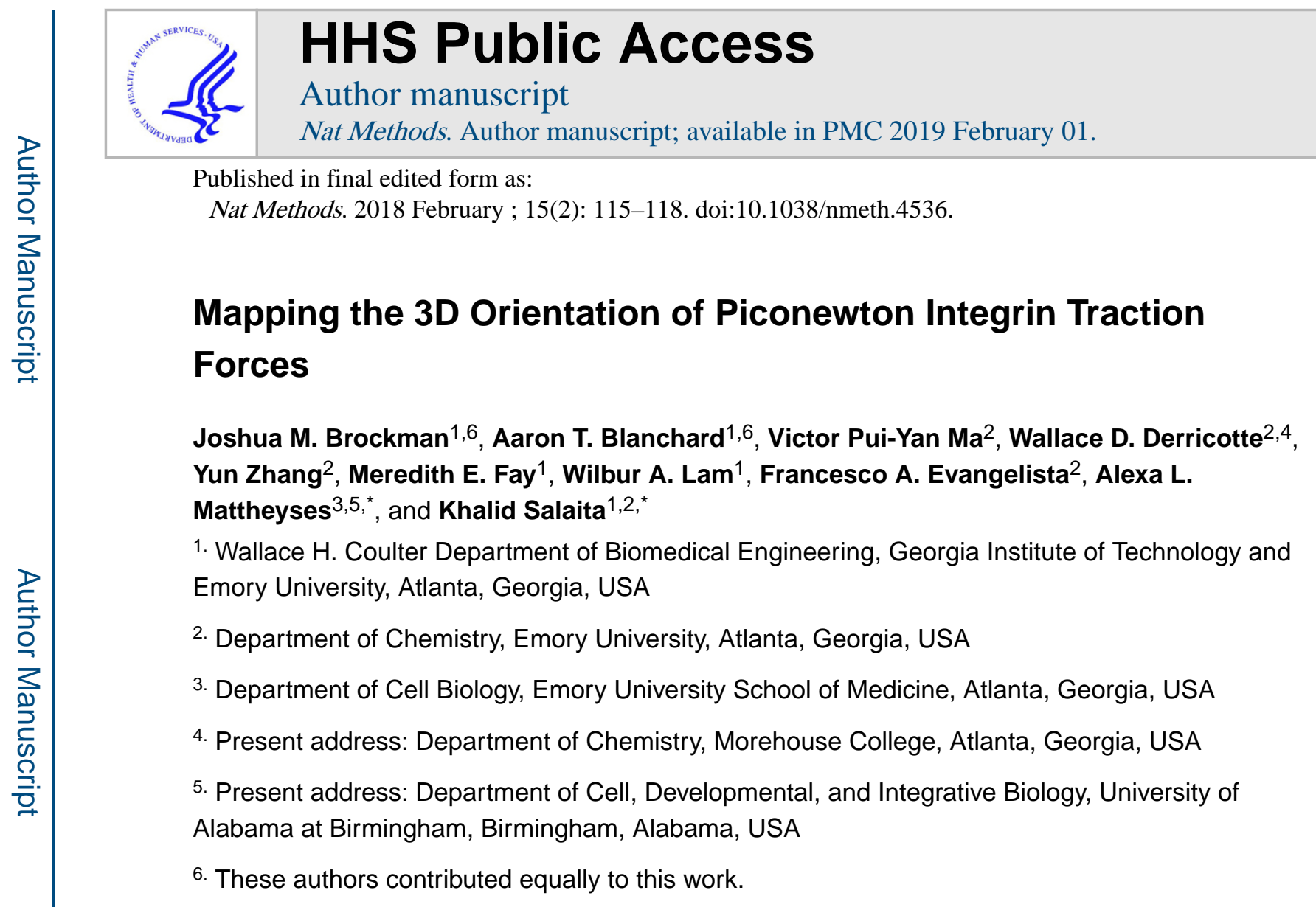

\begin{abstract}
Mechanical forces are integral to many biological processes; however, current techniques cannot map the magnitude and direction of piconewton molecular forces. Here, we describe molecular force microscopy, leveraging molecular tension probes and fluorescence polarization microscopy to measure the magnitude and 3D orientation of cellular forces. We mapped the orientation of integrin-based traction forces in mouse fibroblasts and human platelets, revealing alignment between the organization of force-bearing structures and their force vectors.
\end{abstract}

\title{
Editorial summary:
}

Molecular force microscopy employs a combination of fluorescence polarization microscopy and molecular tension sensors to determine the orientation of cellular forces. The technology is demonstrated for integrin-mediated forces in platelets and fibroblasts.

Cell mechanics regulate many biological processes including immune recognition, coagulation, cell migration, and differentiation. The gold standard for mapping the direction and magnitude of cell forces, traction force microscopy (TFM) relies on measuring the cell-

\footnotetext{
*Correspondence should be addressed to K.S. (k.salaita@emory.edu) or A.L.M. (mattheyses@emory.edu). Author Contributions: A.L.M. and K.S. conceived the general principle of MFM. J.M.B., A.T.B., A.L.M., and K.S. conceived of applying excitation resolved fluorescence microscopy. J.M.B., A.T.B., A.L.M., and K.S. designed experiments. J.M.B. performed experiments. J.M.B. performed anisotropy simulations. A.T.B. derived tilt angle measurement and performed error simulations. J.M.B., Y.Z., and V.M. synthesized reagents and prepared DNA-modified surfaces. J.M.B. and A.T.B. performed data analysis and wrote the required programs. J.M.B., M.E.F., and W.A.L. designed platelet aggregate experiments. M.E.F. assisted with platelet handling. W.D.D. and F.A.E. calculated the Cy3B transition dipole moment. J.M.B., A.T.B., A.L.M., and K.S. wrote the manuscript. Competing Financial Interests: The authors declare no competing financial interests.
} 
driven deformation of polymer substrates loaded with fluorescent beads ${ }^{1,2}$. Although widely used, TFM provides $\mu \mathrm{m}$ spatial resolution and $\mathrm{nN}$ force sensitivity, orders of magnitude greater than the $\mathrm{pN}$ forces applied by individual receptors.

To address these limitations, we previously developed molecular tension probes to map the magnitude of receptor forces with $\mathrm{pN}$ sensitivity ${ }^{3}$. Molecular tension probes are comprised of an extendible "spring-like" element (e.g. polyethylene-glycol, DNA, or protein), flanked by a fluorophore and quencher and immobilized on a surface. Receptor forces applied to the probe extend the "spring," separating the fluorophore-quencher pair, and generating up to a 100 -fold increase in fluorescence ${ }^{4,5}$. Complementary approaches employing genetically encoded FRET-based tension probes are powerful ${ }^{6}$, but also lack information on force orientation ${ }^{2}$. Indeed, no current method can map the orientation of traction forces with $\mathrm{pN}$ sensitivity. Herein, we integrate fluorescence polarization (FP) techniques with molecular tension probes to enable molecular force microscopy (MFM), thereby generating the highest resolution maps of $\mathrm{pN}$ traction force orientations.

To measure force orientation, we applied FP to DNA-based tension probes labeled with Cy3B (Supplementary Fig. 1, Supplementary Table 1-2) because this class of probes displays the highest signal-to-background ratio of reported molecular force probes ${ }^{5}$, a tunable force response threshold ${ }^{4}$, and cyanine dyes stack against the nucleobases of the duplex, perpendicular to its long axis ${ }^{7}$. Stacking is vital, because FP requires a known and fixed dye orientation relative to the biomolecule to determine its orientation. When receptormediated forces exceed the $\mathrm{F}_{1 / 2}$, the equilibrium force at which $50 \%$ of hairpins unfold, the dye separates from the quencher, producing a significant enhancement in fluorescence (Fig. 1a). Importantly, rotatable bonds anchoring the probe enable the DNA and attached fluorophore to explore a hemisphere of orientations (grey hemisphere, Fig 1b). Receptor forces dictate the orientation of the DNA probe, restricting both its mobility (Supplementary Note 1, Supplementary Fig. 2) and the allowable conformations of the cyanine dye (yellow disc, Fig. 1b). Moreover, hairpins experiencing $\mathrm{F}<\mathrm{F}_{1 / 2}$ are folded and do not contribute to the fluorescence signal; in contrast hairpins experiencing $\mathrm{F} \Psi_{1 / 2}$ generate the FP signal. Accordingly, MFM is extraordinarily sensitive due to this "mechano-selection", where the random orientations of relaxed probes do not dampen the FP signal as is the case for conventional polarization measurements, and only mechanically strained and oriented probes generate a FP response.

We first tested our ability to measure the orientation of traction forces generated by integrin $a_{I I b} \beta_{3}$ in human platelets because their activation and clotting functions are mediated by mechanical forces ${ }^{8-10}$. Human platelets seeded on tension probes presenting the RGD peptide (Arg-Gly-Asp) produced robust signal, corresponding to integrins that apply $\mathrm{F} \geq 4.7$ $\mathrm{pN}\left(\mathrm{F}_{1 / 2}\right)$ (Fig. 1c) ${ }^{4}$. When these platelets were imaged using emission-resolved $\mathrm{FP}^{11}$, we observed high anisotropy along the axis perpendicular to the excitation polarization and low anisotropy along the parallel axis (Fig. 1c, Supplementary Fig. 3a, 4). Probes linking the Cy3B to the DNA with a flexible linker or probes tagged using Alexa488, which poorly stacks with the DNA, attenuated the anisotropy signal, further confirming the results (Supplementary Fig. 4). Modeling showed that the anisotropy map was consistent with 
forces orienting DNA probes inward towards the cell center (Supplementary Fig. 4, Supplementary Note 2).

To determine the 3D orientation of unfolded DNA probes, we used excitation-resolved FP (Supplementary Fig. 3b), where fluorescence intensity is quantified as a function of excitation polarization $\left(\Phi_{\text {excitation }}\right)^{12}$. This approach only requires adding a rotatable halfwave plate into the path of a polarized excitation source in a conventional fluorescence microscope. Excitation-resolved FP can resolve dye orientation because fluorescence intensity depends on the alignment between the fluorophore and $\Phi_{\text {excitation }}$ (Fig. 1d). Due to the free rotational motion of the DNA helix along its long axis, the stacked fluorophore adopts a plane of orientations (yellow disc, Fig. 1b). By varying $\Phi_{\text {excitation }}$ and measuring total fluorescence, we determined the projection of the dye's orientation onto the imaging plane (Fig. 1b, d), providing the in-plane force orientation $\left(\Phi_{\text {force }}\right)$ and tilt angle ( $\left.\theta_{\text {force }}\right)$ (Supplementary Note 3, Supplementary Fig. 5). We created an interactive graphical interface (Supplementary Software 1) to illustrate the relationship between probe orientation and fluorescence intensity as a function of $\Phi_{\text {excitation}}$. Fluorescence intensity varies sinusoidally with $\Phi_{\text {excitation }}$ (Fig. 1d): $\Phi_{\text {force }}$ is calculated from the phase, while $\theta_{\text {force }}$ is calculated indirectly from the amplitude (Fig. 1d, Supplementary Note 3, Supplementary Fig. 5, Supplementary Table 3-4). We measured the probe density as $694 \pm 32$ molecules $/ \mu \mathrm{m}^{2}$, with an average of 3.4 probes/pixel. Therefore, $\theta_{\text {force }}$ and $\Phi_{\text {force }}$ represent the average receptor force orientation within each diffraction-limited area (Supplementary Note 4 discusses ensemble averaging). In silico modeling showed that the accuracy of MFM depends on the signal brightness and on $\theta_{\text {force. }}$ In Fig. 1, we report force orientations with an error $<15^{\circ}$ for $\theta_{\text {force }}$ and $<10^{\circ}$ for $\Phi_{\text {force, }}$ except for vertical forces $\left(\theta_{\text {force }}<10^{\circ}\right)$, where MFM less accurately measures $\Phi_{\text {force }}$ (Supplementary Note 4, Supplementary Fig. 6, 7). We confirmed the robustness of our approach by measuring the orientation of cyanine dye (DiI)-doped phospholipid membranes (Supplementary Fig. 8).

During an MFM experiment, we acquired images of human platelets on tension probes while continuously rotating the excitation polarization. Representative images at $\Phi_{\text {excitation }}=0^{\circ}$ and $90^{\circ}$ are anti-correlated, confirming that Cy3B was oriented by platelet forces (Fig. 1e). We fit the fluorescence intensity as a function of $\Phi_{\text {excitation }}$ to sinusoids for each pixel to determine the 3D force orientation, producing an MFM map of platelet forces (Fig. 1f-g, Supplementary Fig. 9, Supplementary Software 2). Dipole orientation represents the $\mathrm{XY}$ force axis $\left(\Phi_{\text {force }}\right)$, color represents $\theta_{\text {force, }}$, and dipole length specifies the fraction of unfolded probes. Strikingly, this platelet exhibited force orientation toward an axis rather than isotropic contraction (Fig. 1h). Multi-cell analysis revealed two platelet populations: one with forces oriented toward an axis, the other with forces radially oriented, consistent with previously reported isotropic contraction ${ }^{9}$ (Fig. 1i, Supplementary Fig. 10). Recent evidence links microtubule marginal band extension during platelet activation with microtubule coiling, and formation of a symmetry axis ${ }^{13}$, providing a potential explanation for the observed force axis. Platelet immunostaining revealed that tension and actin flank a coiled microtubule band (Supplementary Fig. 11). Forces at the platelet edge were radially isotropic, but interior forces exhibited axial organization, indicating two distinct modes of contraction during platelet activation (Supplementary Fig. 12). 
Many biological processes require coordination between cells ${ }^{14}$; however, TFM resolution is reduced when imaging forces in multicellular structures ${ }^{15}$. MFM addresses this issue because each probe reports force orientation without mechanical interference from adjacent receptors or cells. To demonstrate this capability, we applied MFM to platelet aggregates, determining that the average force axes of the constituent platelets did not appear to be ordered across platelet aggregates ( 6 aggregates with $>5$ cells from $n=3$ independent experiments) (Fig. 2a-c, Supplementary Fig. 9). Force orientations were not aligned in $38 \%$ (12/31) of platelet-platelet boundaries (Fig. 2c, inset 2), further indicating that platelet forces are not necessarily coordinated within a clot. These findings, combined with the observation that platelet contraction increases with microenvironment stiffness ${ }^{8}$, could contribute to understanding the characteristic anisotropy and spatial heterogeneity of clot structure and mechanics ${ }^{16}$.

To further demonstrate the power of MFM, we mapped the orientation of integrin-mediated forces in NIH-3T3 fibroblasts. Fibroblasts adhere through integrin-based focal adhesions (FAs), which physically bridge the cytoskeleton with the extracellular matrix. Stablytransfected GFP-paxillin fibroblasts were plated on the tension probes and imaged, generating an MFM map (Fig. 2d-g). The integrin forces were generally radially aligned (Fig. 2g, Supplementary Fig. 9, 13). We identified 494 FAs by segmenting the tension images of 15 cells. Within individual FAs, $\Phi_{\text {force }}$ values were coherent (Supplementary Fig. 13) and tilt angles displayed only $1.5^{\circ}$ of variation between the distal and proximal sides of the adhesion (Supplementary Fig. 13), indicating that integrin forces were aligned both inplane and normal to the substrate.

Next, we compared integrin MFM maps for platelets and fibroblasts and found the average $\theta_{\text {force }}$ was similar at $40^{\circ} \pm 2^{\circ}$ and $39^{\circ} \pm 4^{\circ}$, respectively. Fibroblast forces are more vertical at the cell center, with a median $\theta_{\text {force }}$ of $30^{\circ}$, and become increasingly lateral near the cell periphery with median $\theta_{\text {force }}$ of $41^{\circ}$. In comparison, platelets exhibit less variation in $\theta_{\text {force }}$ from the cell center (median $35^{\circ}$ ) to its periphery (median $40^{\circ}$ ) (Supplementary Fig. 14).

In summary, MFM revealed a previously unreported axis of force alignment in activated platelets ${ }^{9}$. Additionally, MFM directly measures platelet integrin forces. Unlike TFM techniques, MFM does not require advanced computational capabilities to resolve the full $3 \mathrm{D}$ vector of cellular forces ${ }^{17}$. Advanced TFM measurements of mature FAs suggested a sizable proportion of cell traction was normal to the substrate plane ${ }^{17}$ in agreement with our measured $\theta_{\text {force }}$ of $\sim 40^{\circ}$ in fibroblast FAs. Structural analyses of FAs using super-resolution imaging (iPALM) and scanning angle fluorescence interference contrast microscopy report tilt angles for the FA adaptor talin, of 50-60 18,19 . MFM reports integrin forces within $\sim 10^{\circ}$ of the talin average tilt angle, providing a direct link between molecular forces and FA structure. Note that because MFM reports ensemble force orientations, the measured tilt angle provides a lower bound on $\theta_{\text {force }}$ for individual receptor forces (Supplementary Note 4). Additionally, GFP- $a_{v} \beta_{3}$ integrin emission-resolved FP measurements showed coalignment of integrins within $\mathrm{FAs}^{20}$, consistent with our measurements showing low variance of integrin forces within FAs. Springer and colleagues have argued that forces lateral to the cell membrane are responsible for activating integrins, in contrast to normal forces that drive the inactive state of the receptor ${ }^{21}$. MFM provides the capabilities to 
experimentally test this hypothesis. Due to its simplicity and compatibility with fluorescence microscopy, we anticipate MFM will provide a powerful link between structural biology and mechanobiology.

\section{Online Methods}

\section{Materials}

N-hydroxyl succinimide azide (NHS-azide,88902), DiI (CN-1006) and NHS-Biotin (20217) were purchased from Thermo Fischer Scientific (Waltham, MA). Cy3B-NHS ester (PA63101) was acquired from GE Healthcare Life Sciences (Pittsburgh, PA). DNA was custom synthesized by Integrated DNA Technologies (Coralville, IA). Cyclo[Arg-Gly-Aspd-Phe-Lys(PEG-PEG)] (PCI-3696-PI), elsewhere abbreviated as cRGD, was acquired from Peptides International (Louisville, KY). \#1.5 25mm glass coverslips (Product code: 722501) were purchased from Electron Microscopy Sciences (Hatfield, PA). Streptavidin (S00001) was obtained from Rockland-Inc(Pottstown, PA). $\mu$-Slide VI ${ }^{0.4} 6$ channel slides (80606) and $25 \mathrm{~mm} \times 75 \mathrm{~mm}$ glass coverslips (10812) were purchased from Ibidi (Verona, WI). 96well glass bottom plates (655892) were obtained from Greiner (Kremsmünster, Austria). 5$\mu \mathrm{m}$ silica beads (SS06N) were acquired from Bang Laboratories (Fishers, IN). Lipoic acidPEG-NHS (Mw=3400, PG2-LANS-3k) and mPEG-NHS (Mw=2000, PG1-TH-2k) were purchased from Nanocs (New York, NY). 1,2-dioleoyl-sn-glycero-3-phosphocholine (DOPC) lipid was purchased from Avanti Polar Lipids Inc. (Alabaster, AL). Gold nanoparticles were acquired from Nanocomposix (San Diego, CA). TEM performed by Nanocomposix indicated an average gold nanoparticle diameter of $8.6 \pm 0.6 \mathrm{~nm}$. All other reagents and materials (unless otherwise stated) were purchased from Sigma-Aldrich and used without purification. All buffers were prepared with $18.2 \mathrm{M} \Omega$ nanopure water.

\section{Methods}

Streptavidin Surface Preparation-DNA-based tension probes were prepared based on our published protocols ${ }^{4,5}$. Briefly, glass coverslips were successively sonicated ( $\left.\sim 5 \mathrm{~min}\right)$ in nanopure $\left(18.2 \mathrm{M} \Omega\right.$ ) water followed by sonication in ethanol. Coverslips were dried at $90^{\circ} \mathrm{C}$ for 15 minutes and then cleaned in piranha solution, a 3:1 mixture of concentrated sulfuric acid and 30\% hydrogen peroxide (caution, piranha acid is extremely corrosive and may explode if exposed to organic materials), washed 6 times in nanopure water and then immersed in 4 successive beakers of ethanol. The piranha-cleaned surfaces were reacted with 2.5\% 3-(Aminopropyl)triethoxysilane (APTES) $\mathrm{v} / \mathrm{v}$ in ethanol in a fume hood for $1 \mathrm{~h}$. The coverslips were then washed 3 times with ethanol and dried under a stream of ultra-high purity $\mathrm{N}_{2}$. Immediately following drying, the surfaces were cured in an oven at $90^{\circ} \mathrm{C}$ for 30 min. After cooling, the surfaces were incubated overnight with NHS-Biotin (2-3 mg ml$\left.{ }^{-1}\right)$ in DMSO. The surfaces were then washed with ethanol, dried under ultra-high purity $\mathrm{N}_{2}$, mounted in Attofluor Chambers (Life Technologies), and washed with $10 \mathrm{~mL}$ of 1x PBS. Surfaces were blocked with $0.1 \%$ BSA (w/v) for 30 min and then washed with $1 x$ PBS. The surfaces were then incubated in $1 \mathrm{mg} \mathrm{mL}^{-1}$ streptavidin in 1x PBS for $45 \mathrm{~min}$. The surfaces were then washed with $10 \mathrm{~mL}$ of $1 x$ PBS. Finally, the surfaces were incubated with $10 \mathrm{nM}$ DNA tension probes for $1 \mathrm{~h}$ and then washed with $10 \mathrm{~mL} 1 \mathrm{x}$ PBS prior to beginning cell experiments. 
Gold Nanoparticle Surface Preparation-Nanoparticle-based tension probes were prepared following our previous work (cite relelvant work). Briefly, rectangular glass coverslips $(25 \times 75 \mathrm{~mm})$ were cleaned using piranha solution as described above. Slides were then washed in 6 successive beakers of nanopure water and then etched in a beaker of $\mathrm{KOH}(0.5 \mathrm{M})$ for 1 hour in an ice-filled sonicator. The coverslips were washed in 6 successive beakers of nanopure water, followed by 3 successive beakers of ethanol. In a fourth beaker of ethanol, slides were reacted with 3\% APTES v/v for $1 \mathrm{~h}$. Coverslips were washed $2 \mathrm{x}$ with acetone, 3-6 times with nanopure water, dried under a stream of $\mathrm{N}_{2}$ gas, and firmly attached to Ibidi channels ( $\mu$-Slide $\mathrm{VI}^{0.4}$ ). The silanized slides were reacted with $1 \%$ w/v lipoic acid polyethylene glycol (MW = 3400) and 10\% w/v 2000 molecular weight polyethylene glycol $\left(\mathrm{PEG}_{2000}\right.$ ) in $0.1 \mathrm{M} \mathrm{NaHCO} 3(\mathrm{pH} 9)$ for $30 \mathrm{~min}$. Slides were washed $3 \mathrm{x}$ with nanopure water and dried under a stream of ultra-high purity $\mathrm{N}_{2}$. Slides were reacted for 30 min with $1 \%$ NHS-acetate in $0.1 \mathrm{M} \mathrm{NaHCO}_{3}(\mathrm{pH} 9)$ to consume any unreacted amines on the surface. $50 \mu \mathrm{L}$ of $8.8 \mathrm{~nm}$ gold nanoparticles (AuNPs), concentration $20 \mathrm{nM}$, were added to each well and allowed to incubate for $30 \mathrm{~min}$. Unbound AuNPs were removed by washing the channels 3 times with $1 \mathrm{~mL}$ of nanopure water. Finally, $45 \mu \mathrm{L}$ of $300 \mathrm{nM}$ DNA tension probes and $2.7 \mu \mathrm{M}$ passivating ssDNA (AuNP anchor strand, Supplementary Table 1) were added to each channel and each channel was sealed firmly with parafilm. Slides were incubated with the DNA overnight at $4^{\circ} \mathrm{C}$ in a petri dish. A water soaked kimwipe was also placed in the petri dish to maintain humidity during this reaction. Just prior to imaging, channels were washed with a cell imaging media and cells were added.

Preparation of small unilamellar vesicle (SUV).-Small unilamellar vesicles with an average diameter of $100 \mathrm{~nm}$ were prepared by lipid extrusion. In brief, $4 \mathrm{mg} \mathrm{mL}^{-1}$ of DOPC lipid was diluted in $\sim 1 \mathrm{~mL}$ chloroform in a round bottom flask and the chloroform was removed by rotary evaporation to form a thin lipid film. The lipid film was further dried under a steam of $\mathrm{N}_{2}$ and then hydrated with $2 \mathrm{~mL}$ of milli-Q water. To dissolve the lipid film, three freeze-thaw cycles were performed. The lipid solution was then repeatedly extruded ( 10 times) through a $100 \mathrm{~nm}$ polycarbonate filter until the solution became clear. The extruded SUVs are stored at $4^{\circ} \mathrm{C}$ and used within 4-6 weeks.

\section{Preparation of Dil-doped Phospholipid Membranes Supported on}

Microparticles-100 $\mu \mathrm{L} 1 \mathrm{mg} \mathrm{mL}^{-1} 5 \mu \mathrm{m}$ silica beads were mixed with $100 \mu \mathrm{l}$ of $2 \mathrm{mg}$ $\mathrm{mL}^{-1}$ DOPC vesicles and equilibrated on a rocker for $15 \mathrm{~min}$ at room temperature. The supported lipid bilayer (SLB) beads were purified by 3 successive 5 min spins at 2000 RPM. After each spin, the supernatant was removed and replaced with $1 \mathrm{~mL}$ of 1x PBS. The SLB beads were incubated with $5 \mu \mathrm{M}$ DiI for 15 minutes. The DiI loaded SLB beads were separated from free DiI via 3 successive 5 min spins at 2000 RPM. Once again, the supernatant was removed in between each spin and replaced with $1 \mathrm{~mL}$ of 1x PBS. Finally, SLB beads were placed in clean wells of a coverslip-bottom 96-well plate in 1x PBS.

Matrix-Assisted Laser Desorption/lonization-Time of Flight (MALDI-TOF)—For peptide MALDI-TOF, saturated a-cyano-4-hydroxycinnaminic acid matrix was prepared in $50 \%$ acetonitrile and $0.1 \%$ trifluoroacetic acid. $1.5 \mu \mathrm{L}$ of the matrix was mixed on a MALDI plate with $1 \mu \mathrm{L}$ of the sample suspended in nanopure water. For DNA MALDI-TOF, a 
matrix consisting of saturated 3-hydroxypicolinic acid in 50\% acetonitrile, $0.1 \%$ trifluoroacetic acid, and $50 \mathrm{mg} \mathrm{mL}^{-1}$ ammonium citrate was prepared. $1.5 \mu \mathrm{L}$ of the matrix was mixed on a MALDI plate with $1 \mu \mathrm{L}$ of the sample in nanopure water. Samples were dried for 20-30 minutes and then analyzed by MALDI-TOF (Voyager STR).

Hairpin Hybridization-DNA oligonucleotides were hybridized at $200 \mathrm{nM} \mathrm{(300} \mathrm{nM} \mathrm{for}$ AuNP-based probes) in a $0.2 \mathrm{~mL}$ Thermowell Tube. DNA was heated to $90^{\circ} \mathrm{C}$ and then cooled at a rate of $1.3^{\circ} \mathrm{C}$ per minute to $35^{\circ} \mathrm{C}$.

Platelet Handling-Experiments were performed with blood from human volunteers drawn according to IRB-approved protocols (Georgia Institute of Technology: Central Institutional Review Board; Emory University, Institutional Review Board) in compliance with all ethical regulations. Informed consent was obtained from all subjects prior to experimentation.

Venous blood was drawn from the arm of human volunteers in two $3 \mathrm{~mL}$ portions with the first portion discarded. Anticoagulant citrate dextrose (ACD) solution $(0.75 \mathrm{~mL})$ was added to the whole blood. The whole blood/ACD mixture was spun at $150 \mathrm{RCF}$ for 15 minutes at the lowest centrifuge acceleration and braking settings to avoid platelet activation. The platelet rich plasma was removed, combined with $10 \%$ ACD v/v, and spun at $900 \mathrm{RCF}$ for 5 minutes at the lowest centrifuge acceleration and braking settings. The platelet poor plasma was aspirated following the spin and the platelets were gently suspended in $2 \mathrm{~mL} \mathrm{1x}$ Tyrodes with $0.1 \%$ BSA w/v. Platelets were allowed to return to a resting state for $\sim 1$ hour prior to the start of each experiment.

Platelet Aggregate Formation-Immediately prior to imaging, platelets were suspended in $1 \mathrm{x}$ Tyrodes ( $\mathrm{pH}$ 7.4) supplemented with $0.1 \%$ BSA w/v, Hank's Balanced Salt Solution, 2 $\mathrm{mM} \mathrm{MgCl} 2,1 \mathrm{mM} \mathrm{CaCl} 2,1$ unit/ $\mu \mathrm{L}$ thrombin, and $10 \mu \mathrm{g} / \mu \mathrm{L}$ fibrinogen. This mixture was inverted 3 times and immediately pipetted into one well of a microchannel formed from an Ibidi $\mu$-slide $\mathrm{VI}^{0.4}$ and a $25 \mathrm{~mm} \times 75 \mathrm{~mm}$ glass coverslip. Platelet aggregates were observed on the surface within 5-10 min.

Immunostaining-Platelets were allowed to spread on RGD-DNA surfaces for $20 \mathrm{~min}$ then fixed for 10 minutes with cold $4 \%$ formaldehyde v/v in 1x PBS. Platelets were permeabilized for 10 min with $0.25 x$ Triton and blocked with $1 \%$ BSA for 40 min. Platelets were stained for actin with Alexa-647 labeled phalloidin (ThermoFisher, A22287) and tubulin with mouse anti bovine a tubulin (ThermoFisher, A21371) followed by an Alexa-488 labeled goat anti-mouse secondary (ThermoFisher, A-21121) following the manufacturer's protocol.

Emission-Resolved Fluorescence Polarization Imaging-Imaging was accomplished on a Nikon Eclipse Ti microscope, operated by Nikon Elements software, a 1.49 NA CFI Apo 100x objective, perfect focus system, and a TIRF laser launch with 80 $\mathrm{mW} 561 \mathrm{~nm}$ and $488 \mathrm{~nm}$ lasers. A Chroma quad cube (ET-405/488/561/640 nm Laser Quad Band) and reflection interference contrast microscopy (RICM) (Nikon: 97270) cube were used for imaging. Widefield epifluorescence illumination was provided by an X-Cite 120 
lamp (Excelitas). An Andor TuCam system with a wire grid polarizer (Moxtek, Andor: TREMFS-F03) split the fluorescence by polarization to two Andor iXon Ultra 897 electron multiplying charge coupled devices. To flip the laser excitation polarization, a half wave plate (Thorlabs) mounted on a slider at $45^{\circ}$ was inserted into the light path. The lasers were operated in widefield mode, focused at the back focal plane and travelling along the optical axis. This optical configuration was used in conducting the experiments depicted in Figure 1c, Supplementary Figure 4, and Supplementary Figure 8b.

Live human platelets imaging was completed within $8 \mathrm{~h}$ of platelet harvesting. Camera background signal was measured from images of 1x PBS. An illumination correction image was produced during each imaging session by averaging 10 images of cy3B in solution. The fluorescence emission was split into components parallel and perpendicular to the laser by a polarizing beam splitter. The polarization bias of the microscope, or G-factor, was computed by taking the ratio of the parallel and perpendicular fluorescence of fluorescein in solution. Large-numerical aperture corrections ${ }^{22}$ were used to correct for the polarization mixing of the microscope.

Excitation Resolved Fluorescence Polarization Imaging-Fluorescence azimuth experiments were conducted on a Nikon Eclipse Ti microscope, operated by Nikon Elements software, with a Chroma quad cube and an RICM cube. A $150 \mathrm{~mW}$ OBIS $561 \mathrm{~nm}$ laser was used to illuminate the sample, operating at $3 \%$ power. The polarization of the laser was rotated via a 400-800 nm SM1-threaded mounted 1-inch achromatic half wave plate (Thorlabs) rotated via a motorized precision rotation stage (Thorlabs, PRM1Z8) and a kcube brushed DC servo motor controller (Thorlabs, KDC101) to control the excitation polarization. The rotator was operated by the Kinesis software. Images were taken on a Hamamatsu ORCA-Flash4.0 v2 Digital CMOS (Product code: C11440-22CU). Images were collected with a $100 \times 1.49$ NA CFI Apochromat TIRF series oil-immersion objective (Nikon Instruments). The exposure time for all imaging experiments was $50 \mathrm{~ms}$. Fluorescence was binned $2 \times 2$ on the CMOS for fibroblast and platelet aggregate experiments to increase the signal-noise ratio. Nikon Elements "Fast Acquisition" was used to maximize the acquisition speed and eliminate any delay between frames. The lasers were operated in widefield mode, focused on the back focal plane and travelling along the optical axis. This optical configuration was used in the acquisition of all data except the data presented in Figure 1c, Supplementary Figure 4 and Supplementary Figure 8b. Prior to image acquisition, the half wave plate was set in motion. After the rotator reached its maximum velocity of $25 \%$ s (corresponding to $50 \%$ s of excitation polarization rotation), 73 fluorescence images were acquired continuously with a $50 \mathrm{~ms}$ exposure time. The entire image sequence required 3.6 seconds to complete. Each image corresponded to a change in excitation polarization of $2.5^{\circ}$ of arc, centered around the target excitation angle (e.g. the polarizer rotated through an arc from $-1.25^{\circ}-1.25^{\circ}$ during the $50 \mathrm{~ms}$ acquisition for the " 0 " " image.

Immediately prior to imaging, platelets were suspended in $\mathrm{pH} 7.4$ 1x Tyrode's Buffer supplemented with $0.1 \%$ BSA w/v, Hank's Balanced Salt solution, $2 \mathrm{mM} \mathrm{MgCl}_{2}$, and $1 \mathrm{mM}$ $\mathrm{CaCl}_{2}$ and then added to the cRGD-DNA probe modified surface. Platelets began spreading on the cRGD streptavidin surface within 5 min of seeding. 
Platelet aggregates were imaged at room temperature in $1 \mathrm{x}$ Tyrodes $(\mathrm{pH} 7.4)$ supplemented with $0.1 \%$ BSA w/v, Hank's Balanced Salt Solution, $2 \mathrm{mM} \mathrm{MgCl}_{2}, 1 \mathrm{mM} \mathrm{CaCl}_{2}, 1$ unit/ $/ \mathrm{L}$ thrombin, and $10 \mu \mathrm{g} / \mu \mathrm{L}$ fibrinogen. Platelet aggregates were imaged on AuNP surfaces because these surfaces produced more robust signals for the aggregates.

Fibroblasts were imaged on cRGD streptavidin surfaces at $37^{\circ} \mathrm{C}$ and $5 \% \mathrm{CO}_{2}$ in DMEM supplemented with $1 \%$ penicillin-streptomycin $(\mathrm{v} / \mathrm{v})$ and $10 \%$ fetal bovine serum without phenol red.

Image Processing-We performed all image processing in MATLAB 2016a (MathWorks). The code is available as Supplementary Software 2. The bioformats toolbox enabled direct transfer of Nikon Elements image files (.nd2) into the MATLAB environment. To save computational time, we analyzed user-identified ROIs containing cells. Edge finding was accomplished through Chan-Vese edge finding (available on MathWorks File Exchange) $)^{23}$.

\section{Correcting for Microscope Polarization Bias and Laser Illumination Profile-} Images of an autofluorescent plastic slide (Chroma: 92001) under conditions identical to cell imaging conditions enabled correction for the microscope's polarization bias and the uneven illumination of the laser. The autofluorescence of the Chroma slide is not ordered, thus any changes in intensity as a function of polarization must be due to the bias introduced by the microscope. We normalized the set of 73 images to the maximum intensity according to the following equation:

$$
\text { Bias }_{i}=\frac{\text { Bias }_{\text {Image }_{i}-\text { background }_{\text {detector }}}}{\max (\text { Bias })}, \quad i=1, \quad 2, \quad \ldots 73
$$

Where $\max (\mathrm{Bias})$ is the detector background subtracted maximum intensity within the image sequence.

We normalized cell images to this set of normalized illumination correction images as follows:

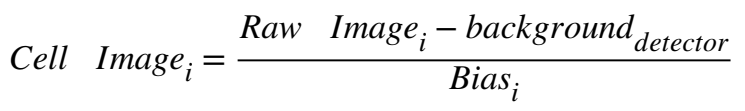

Correcting for both for the uneven illumination profile of the laser and for the polarization bias of the microscope.

Background Subtraction and Signal to Noise Ratio (SNR)-We next determined the local background (background $\mathrm{surface}_{\text {) }}$ ) and subtracted this value from the cellular tension

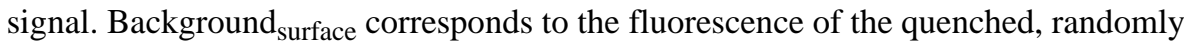
oriented fluorophores attached to DNA probes not experiencing cellular force. 
User-defined ROIs were used to determine the local background intensity. When single cells could be isolated with rectangular ROIs, we defined a background ROI as a "frame" consisting of all pixels within 3 pixels of the edge of the ROI. When the border of the ROI contained fluorescence intensity from a second cell, a user-identified square served in place

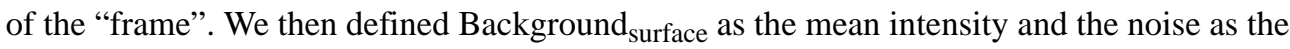
standard deviation of the intensity within the background ROI. Only pixels with a SNR of $>5$ were accepted for analysis.

Photobleaching Correction-To correct for the loss of fluorescence intensity due to

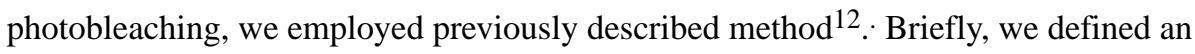
average whole cell bleaching exponent by taking the mean value of the bleach exponents for each pixel of the cell. The bleach exponents for each pixel were calculated as:

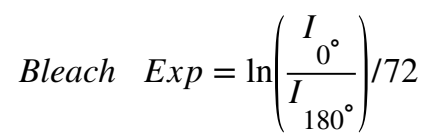

Where $\mathrm{I}_{0^{\circ}}$ is the image corresponding to $\Phi_{\text {excitation }}=0^{\circ}$ and $\mathrm{I}_{180^{\circ}}$ is the image and corresponding to $\Phi_{\text {excitation }}=180^{\circ}$, or Cell Image ${ }_{1 \text {,background subtracted }}$ for $\mathrm{I}_{0^{\circ}}$ and Cell Image $_{73 \text {,background subtracted }}$ for $\mathrm{I}_{180^{\circ}}$ in equation 3. Pixels where fluorescence increased from $\mathrm{I}_{0}$ oto $\mathrm{I}_{180^{\circ}}$ were excluded as this increase must be due to biological changes or to random noise, not photobleaching. To correct for bleaching, we applied the following formula:

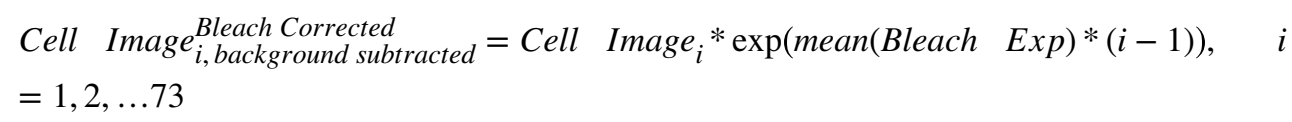

Curve fitting -We thresholded the bleach corrected images by the signal-noise ratio. Because intensity changes as a function of polarization reflect fluorophore orientation, pixel fluorescence intensity will vary from very high to very low as a function of $\Phi_{\text {excitation. }}$. We therefore applied the SNR threshold pixel-by-pixel to the maximum fluorescence intensity in

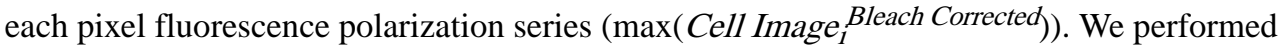
nonlinear curve fitting in MATLAB pixel-by-pixel by fitting fluorescence intensity as a function of $\Phi_{\text {excitation }}$ to the function:

$$
\text { Intensity }=\text { amplitude } * \cos \left(2\left(\phi_{\text {excitation }}-\text { azimuth }\right)\right)+\text { average }
$$


The fitted azimuth gives the average fluorophore orientation, described as the azimuth within a pixel, while the amplitude and average provide the tilt angle of the cellular force (Supplementary Note 3 ). To speed computation, we employed a dynamic initial guess for each pixel: the initial amplitude was set as $1.35 *$ (Imax-average), the average was initially set as the average intensity within each pixel, and the phase was initially specified as the azimuth, defined by:

$$
\text { azimuth guess }=\frac{1}{2} \arctan \left(\frac{I_{45^{\circ}}-I_{135^{\circ}}}{I_{0^{\circ}}-I_{90^{\circ}}}\right)
$$

Hairpin Percent Open Calculation-Because DNA hairpins exhibit near-binary transitions between a fluorescent open state $\left(F_{\text {receptor }}>F_{1 / 2}\right)$ and a quenched closed state $\left(\mathrm{F}_{\text {receptor }}<\mathrm{F}_{1 / 2}\right)$, the fluorescence intensity of a surface is linearly dependent on the percent of probes that are open within a pixel. We prepared both quenched Black Hole Quencher 1 (BHQ-1) labeled immobilization strand) and unquenched (unlabeled immobilization strand) streptavidin and AuNP surfaces as described above. The quenched surface intensity provides a greyscale value for $0 \%$ open DNA hairpins while the unquenched surface provides a greyscale value for $100 \%$ open DNA hairpins. We calculated the dequenching ratio as:

$$
\text { dequenching ratio }=\frac{I_{100 \% \text { open }}-\text { background }_{\text {detector }}}{I_{0 \% \text { open }}-\text { bckground }_{\text {detector }}}
$$

The theoretical maximum of experimental surfaces was then calculated as:

$$
I_{\text {maximum }}=\text { dequenching } \text { ratio } * I_{\text {background }}
$$

The percentage of open hairpins within each pixel was then calculated as:

$$
\% \text { Open }=\frac{I_{\text {pixel }}-I_{\text {background }}}{I_{\text {maximum }}-I_{\text {background }}}
$$

Measuring the Density of DNA Tension Probes on the Surface-A fluorescent lipid bilayer calibration curve was constructed based on a previously reported protocol ${ }^{24}$. Small unilamellar vesicles (DOPC and increasing amounts (0-0.075 mol\%) of Lissamine Rhodamine B-DHPE) were prepared and deposited on base etched, glass bottom 96 well plates for 15 min to form SLBs. Fluorescence intensity was measured using a Nikon Ti-E microscope. The density of fluorophores per unit area was determined from the footprint of DOPC $\left(0.72 \mathrm{~nm}^{2}\right)$ within supported lipid membranes ${ }^{25}$. Therefore, the number of DOPC molecules per $\mu \mathrm{m} 2$ of supported lipid bilayer is $\sim 2.787 \times 10^{6} / \mu \mathrm{m} 2$. We constructed a supported lipid bilayer calibration curve to relate the known Lissamine Rhodamine B-DHPE density to fluorescence intensity. To use this calibration curve to calculate Cy3B-DNA 
density, a scaling factor ( $F$ factor) is introduced to account for the difference in brightness between Cy3B and Rhodamine B. We prepared varying concentrations (50, 100, and 200 $\mathrm{nM}$ ) of Cy3B-DNA and Lissamine Rhodamine B-DHPE molecules and compared their intensities at the same concentration to obtain the $F$ factor, defined as:

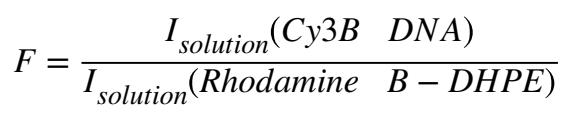

where $I_{\text {solution }}$ (Cy3B-DNA) and $I_{\text {solution }}$ (Rhodamine B-DHPE) are the intensity of the Cy3B-DNA or the Rhodamine B-DHPE in solution at identical concentrations, measured on a fluorescence microscope by focusing into the solution ( 10 $\mu \mathrm{m}$ above the coverslip). We obtained an average $F$ factor of $\sim 12.47$.

We measured the average intensity (from 30 ROIs) of an "unquenched" Cy3B-DNA tension probe surface as $10356 \pm 329$ A.U. (mean \pm s.e.m.). We then converted this intensity into the equivalent Lissamine Rhodamine B-DHPE intensity. Finally, we used our Lissamine Rhodamine B-DHPE calibration curve to estimate the Cy3B-DNA tension probe density as $694.6 \pm 32.7$ probes per $\mu \mathrm{m} 2$ (mean \pm s.e.m.) as specified in a previous report ${ }^{24}$.

Generating an MFM Map-Force orientation was calculated for every pixel in the cell images that exceeded a minimum signal-noise value. The tilt angle was calculated for each pixel as described in Supplementary Note 3. The percent of open MFM hairpins within each pixel was calculated from equation 10 based on the fitted maximum intensity (average +amplitude, calculated from the curve fitting detailed in equation 6). Dipoles were plotted via Matlab's "quiver" function. Vector length was scaled by the percent of open hairpins. Dipole color was specified by tilt angle.

Accounting for Hardware/Software Delays-Delays in Nikon Elements and/or in the Kinesis software caused image acquisition to begin at an excitation polarization slightly offset from $0^{\circ}$. To account for these delays, we measured the azimuth of DiI-doped SLB's on a $5 \mu \mathrm{m}$ bead. We observed a constant offset of approximately $14^{\circ}$ between tangents to the bead surface and the measured DiI orientation. We validated the measured offset by applying it to bead datasets not used in the calculation of the offset parameter, producing the measured dipoles tangential to the bead surface in Supplementary Fig 8. This $14^{\circ}$ offset was applied to all subsequent MFM acquisitions.

\section{Synthesis}

DNA MFM probes were synthesized as previously described ${ }^{4}$. The sequences of all strands are provided in Supplementary Table 1. Briefly, 100 nmoles of c(RGDfK(PEG-PEG)) was reacted with approximately 150 nmoles of NHS-azide in DMF overnight. Product 1 was purified via reverse phase HPLC with a Grace Alltech C18 column $(1 \mathrm{~mL} / \mathrm{min}$ flow rate; Solvent A: 0.1M TEAA + 0.05\% TFA, Solvent B: acetonitrile + 0.05\% TFA; starting condition: $90 \% \mathrm{~A}+10 \% \mathrm{~B}, 1 \% / \mathrm{min}$ gradient $\mathrm{B}$ ) and its identity was verified with MALDITOF (Supplementary Fig. 1, Supplementary Table 2). 
Product 1 was ligated to the tension probe ligand strand via 1,3-dipolar cycloaddition reaction. Briefly, 5 nmoles of alkyne ligand strand was reacted overnight with $\sim 75$ nanomoles of product 1 in the presence of $0.5 \mathrm{M}$ ascorbic acid, $0.5 \mathrm{M} \mathrm{Cu}$-TBTA, and 50\% DMSO. The product was purified with a $\mathrm{P} 2$ size exclusion column, and then using reverse phase HPLC with an Agilent Advanced oligo column $(0.5 \mathrm{~mL} / \mathrm{min}$ flow rate; Solvent A: 0.1M TEAA, Solvent B: acetonitrile; starting condition: $90 \% \mathrm{~A}+10 \% \mathrm{~B}, 1 \% / \mathrm{min}$ gradient B) and the product's identity was verified via MALDI-TOF (Supplementary Fig. 1, Supplementary Table 2). Following the above procedure, products 2, 3, and 6 were produced by varying the identity of the alkyne-conjugated ligand strand.

Products 2 and 3 were dried and reacted overnight with a 15x excess of Cy3B-NHS dissolved in $1 \mu \mathrm{L}$ DMSO. The total reaction volume was $10 \mu \mathrm{L}$, composed of $1 \mathrm{x}$ PBS supplemented with $0.1 \mathrm{M} \mathrm{NaHCO}_{3}$. The reaction pot was purified by a $\mathrm{P} 2$ size exclusion gel to remove unreacted dye followed by HPLC purification $(0.5 \mathrm{~mL} / \mathrm{min}$ flow rate; Solvent A: $0.1 \mathrm{M}$ TEAA, Solvent B: acetonitrile; starting condition: $90 \% \mathrm{~A}+10 \% \mathrm{~B}, 1 \% / \mathrm{min}$ gradient B) to purify products 4 and 5 . The identities of products 4 and 5 were confirmed via MALDI-TOF (Supplementary Fig. 1, Supplementary Table 2).

Please note that the DNA probes immobilized using AuNP have been previously characterized ${ }^{5}$ and are not described here.

\section{Calculation of Cy3B Transition Dipole Moment}

All calculations were performed using version 4.0.0 of the $\mathrm{ORCA}^{26}$ software package. Geometry optimization of the molecule was performed using the B3LYP $27-30$ functional and the $6-31 \mathrm{G}^{* 31}$ basis set, while the TDDFT (Time-dependent density functional theory) excited state calculations and geometry optimizations used the $\omega \mathrm{B} 97 \mathrm{X}$ functional ${ }^{32}$ and the correlation consistent polarized valence double zeta (cc-pVDZ) ${ }^{33}$ basis set (Supplementary Fig $4 a)$. The $\omega \mathrm{B} 97 \mathrm{x}$ functional is a range-separated functional designed to handle longrange/charge transfer character of excited state. The optimized geometries for the ground state $\left(S_{0}\right)$ and the excited state $\left(S_{1}\right)$ are shown in Supplementary Tables 3 and 4 , respectively. The TDM of the excited state corresponding to the first absorption peak calculated at the ground state geometry is $\underline{(\mathrm{x}: 12.25, \mathrm{y}: 2.56, \mathrm{z}: 0.08)} \mathrm{D}$, while the TDM corresponding to the first emission peak calculated at the excited state geometry is ( $\mathrm{x}: 11.71, \mathrm{y}: 2.38, \mathrm{z}: 0.15) \mathrm{D}$ (Supplementary Fig. 4b). Solvation effects were taken into account in the ground state geometry optimization using the continuum solvent model $\mathrm{COSMO}^{34}$ (conductor-likescreening model), while the TDDFT calculations accounted for solvation effects using the $\mathrm{PCM}^{35}$ (Polarizable Continuum Model) available in ORCA.

Statistics-Two tailed two-sample t-tests were run on the sample means of the independent experiments for anisotropy differences between different tension probes (Supplementary Figure 4a). The differences in the fitted amplitudes of individual platelets on the different tension probes were quantified by a two tailed two-sample allowing unequal variance. A paired t-test was used to compare the distributions of the central versus ring force distributions in platelets (Supplementary Figure 12g). A two-sample $t$ test allowing unequal variance was also used to compare the whole-cell average platelet and fibroblast tilt 
angles (Supplementary Figure 14a). A one-way ANOVA was used to quantify the statistical significance of the radial dependence of the platelet and fibroblast tilt angles (Supplementary Figure 14b). The Rayleigh test for uniformity was used on the angle-doubled average force axes of platelets within platelet aggregates to determine whether the average force axes were non-uniformly distributed (Figure 2c).

Code Availability-The analysis software and GUI explaining MFM's operating principles are available as Supplementary Software 1 and 2.

Data Availability-The data that support the findings of this study are available from the corresponding authors upon request.

Further information regarding the experimental design may be found in the Life Sciences Reporting Summary.

\section{Supplementary Material}

Refer to Web version on PubMed Central for supplementary material.

\section{Acknowledgments:}

The authors thank A. Garcia (Wallace H. Coulter Department of Biomedical Engineering, Georgia Institute of Technology and Emory University) for providing NIH-3T3 fibroblasts stably transfected with GFP-paxillin, E. Bartle and T. Urner for assistance with optical systems, and Y. Liu and J. Kindt for helpful discussion. The work was supported through NIGMS R01 GM124472 (K.S.), NSF 1350829 (K.S.), NSF CAREER 1553344 (A.L.M.), NSF IDBR 1353939 (K.S. and A.L.M.), NSF GRFP DGE-1444932 (J.M.B., A.T.B. and M.E.F.), NSF CAREER 1150235 (W. A. L.), NIH Grants ROIHL121264 (W.A.L.), ROI HL130918 (W.A.L.), U01-HL117721 (W.A.L.), and U54HL112309 (W.A.L.).

\section{References}

1. Dembo M, Oliver T, Ishihara A \& Jacobson K Biophys J 70, 2008-2022 (1996). [PubMed: 8785360]

2. Polacheck WJ \& Chen CS Nat Methods 13, 415-423 (2016). [PubMed: 27123817]

3. Stabley DR, Jurchenko C, Marshall SS \& Salaita KS Nature Methods 9, 64-U172 (2012).

4. Zhang Y, Ge C, Zhu C \& Salaita K Nat Commun 5, 5167 (2014). [PubMed: 25342432]

5. Liu Y et al. Proc Natl Acad Sci U S A 113, 5610-5615 (2016). [PubMed: 27140637]

6. Grashoff C et al. Nature 466, 263-266 (2010). [PubMed: 20613844]

7. Iqbal A et al. Proc. Natl. Acad. Sci. U. S. A 105, 11176-11181 (2008). [PubMed: 18676615]

8. Qiu Y et al. Proc Natl Acad Sci U S A (2014).

9. Henriques SS, Sandmann R, Strate A \& Koster S Journal of Cell Science 125, 3914-3920 (2012). [PubMed: 22582082]

10. Coller BS \& Shattil SJ Blood 112, 3011-3025 (2008). [PubMed: 18840725]

11. Kampmann M, Atkinson CE, Mattheyses AL \& Simon SM. Nat Struct Mol Biol 18, 643-649 (2011). [PubMed: 21499242]

12. DeMay BS, Noda N, Gladfelter AS \& Oldenbourg R Biophys J 101, 985-994 (2011). [PubMed: 21843491]

13. Diagouraga B et al. J Cell Biol 204, 177-185 (2014). [PubMed: 24421335]

14. Sunyer R et al. Science 353, 1157-1161 (2016). [PubMed: 27609894]

15. Tang X, Tofangchi A, Anand SV \& Saif TA PLoS Comput Biol 10, e1003631 (2014). [PubMed: 24901766] 
16. Weisel JW Biophys Chem 112, 267-276 (2004). [PubMed: 15572258]

17. Legant WR et al. Proc Natl Acad Sci U S A 110, 881-886 (2013). [PubMed: 23277584]

18. Kanchanawong P et al. Nature 468, 580-584 (2010). [PubMed: 21107430]

19. Paszek MJ et al. Nat Methods 9, 825-827 (2012). [PubMed: 22751201]

20. Swaminathan V, et al. Proc Natl Acad Sci U S A 114, 10648-10653 (2017). [PubMed: 29073038]

21. Zhu J et al. Mol Cell 32, 849-861 (2008). [PubMed: 19111664]

22. Axelrod D Biophys J 26, 557-573 (1979). [PubMed: 263688]

23. Chan TF \& Vese LA IEEE Trans Image Process 10, 266-277 (2001). [PubMed: 18249617]

24. Galush WJ, Nye JA \& Groves JT Biophys J 95, 2512-2519 (2008). [PubMed: 18515392]

25. Vacklin HP, Tiberg F \& Thomas RK Biochim Biophys Acta 1668, 17-24 (2005). [PubMed: 15670727]

26. Neese F Comp Mol Sci 2, 73-78 (2012).

27. Becke AD J Chem Phys 98, 1372-1377 (1993).

28. Lee C, Yang W \& Parr RG Phys Rev B Condens Matter 37, 785-789 (1988). [PubMed: 9944570]

29. Vosko SH, Nusair WM Candian J Phys 58, 1200-1211 (1980).

30. Stephens PJ, Devlin FJ, Chablowski CF \& Frisch MJ Journal Phys Chem 98, 11623-11627 (1994).

31. Krishnan R, Binkley JS, Seeger R \& Pople JA J Chem Phys 72, 650-654 (1980).

32. Chai J \& Head-Gordon M Journal Chem Phys 128 (2008).

33. Dunning TH The Journal of Chemical Physics 90, 1007-1023 (1988).

34. Klamt A J Phys Chem 99, 2224-2235 (1995).

35. Marenich AV, Cramer CJ \& Truhlar DG J Phys Chem B 113, 6378-6396 (2009). [PubMed: 19366259] 
a

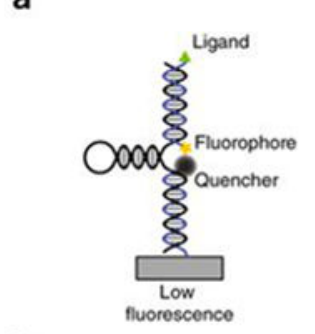

b
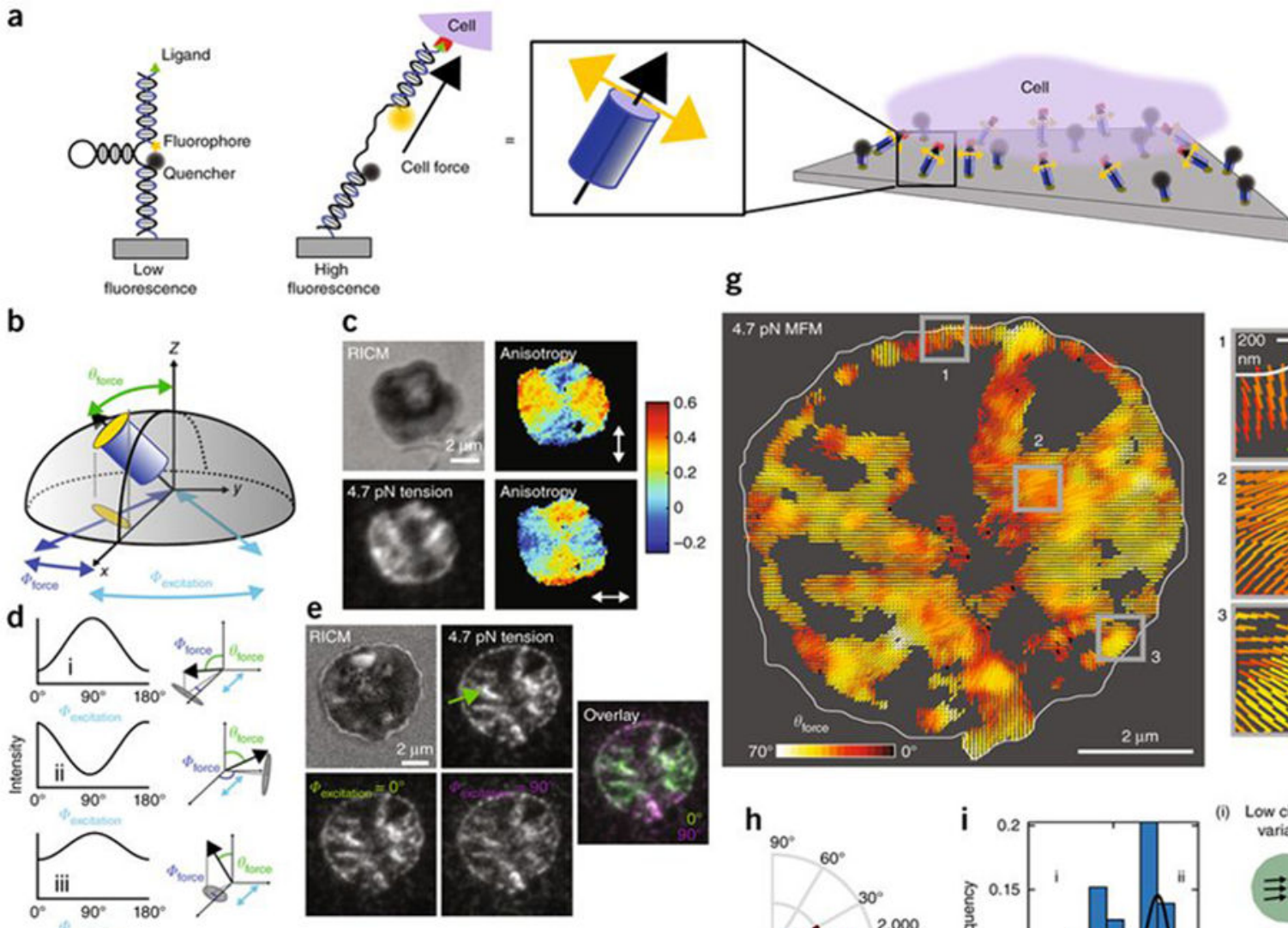

C
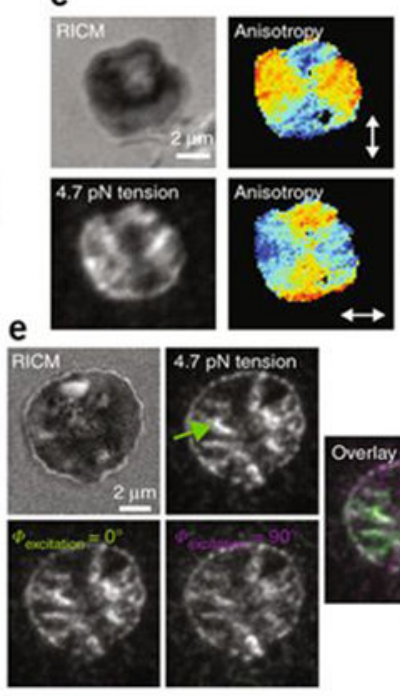

f

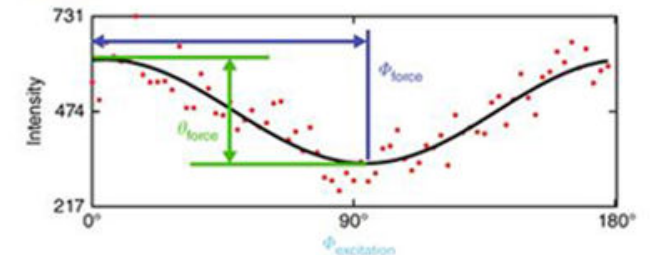

g
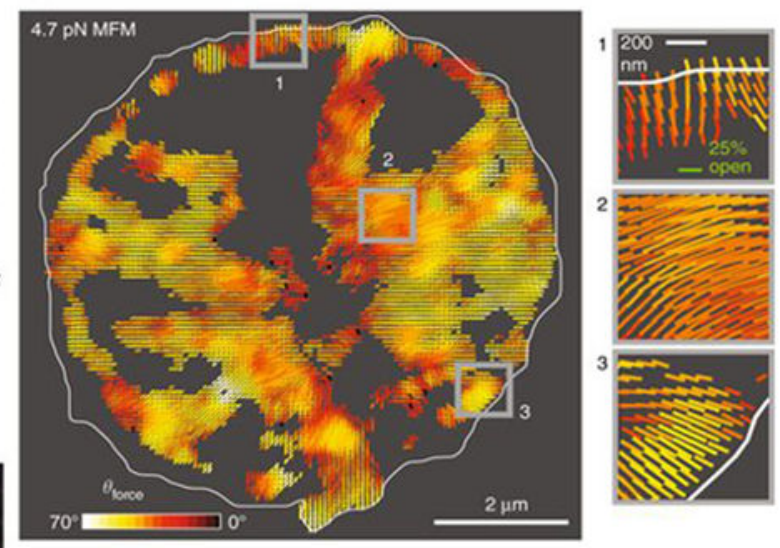

h
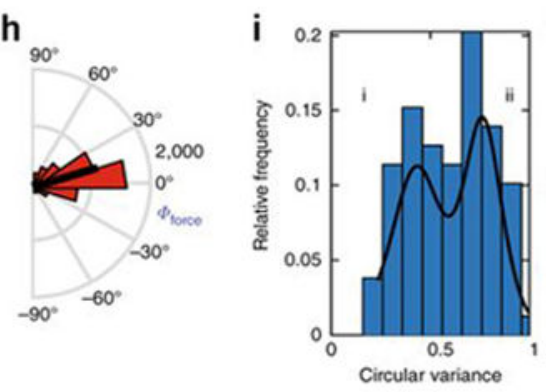

(i) Low circular variance

(ii) High circular variance

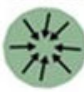

Figure 1: Excitation-resolved fluorescence polarization microscopy enables the measurement of molecular force orientation and magnitude.

(a) Principle of molecular tension probes. Yellow arrows: fluorophore transition dipole moments, green: RGD ligands, red: integrin receptors. (b) Receptor forces dictate DNA probe orientation (grey hemisphere: possible orientations), fluorophore orientation and the $\mathrm{XY}$ projection of Cy3B (yellow ellipse in the XY plane). (c) Reflective interference contrast microscopy (RICM), $4.7 \mathrm{pN}$ tension image, and fluorescence anisotropy (white arrows: excitation polarization) images of a human platelet on a DNA tension probe surface (representative of 107 platelets from $n=4$ independent experiments) (d) Hypothetical plots of fluorescence intensity as a function of $\Phi_{\text {excitation }}$ for three force orientations. Maximum intensity occurs when $\Phi_{\text {excitation }}$ and the fluorophore XY projection (grey ellipse) align. Force XY orientation controls the phase (i-ii), while tilt angle alters amplitude (i-iii). Cyan arrows indicate $\Phi_{\text {excitation }}=0^{\circ}$. (e) Platelet RICM, $4.7 \mathrm{pN}$ total tension, tension at $\Phi_{\text {excitation }}=0^{\circ}$ (green) and $\Phi_{\text {excitation }}=90^{\circ}$ (magenta), and overlay. (f) Fluorescence intensity as a function of $\Phi_{\text {excitation (red dots) for a single pixel (green arrow in (1e)) and sinusoidal fit }}$ (solid line). The $3 \mathrm{D}$ force is described by the phase $\left(\Phi_{\text {force }}\right)$ and the amplitude (force tilt; 
$\left.\theta_{\text {force }}\right)$ (g) MFM map of platelet integrin forces. Dipole orientation: $\Phi_{\text {force }}$, color: $\theta_{\text {force }}$, and length: percentage of open tension probes. Grey background represents intensity below a threshold of signal-noise ratio $<5$. Representative of 79 MFM maps of platelets from $n=5$ independent experiments. (h) Radial histogram of $\Phi_{\text {force }}$ for the platelet in (f). (i) Histogram of circular variance of population platelet XY force projections. Schematics depict the force orientation for the two population of platelets with high and low circular variance. 

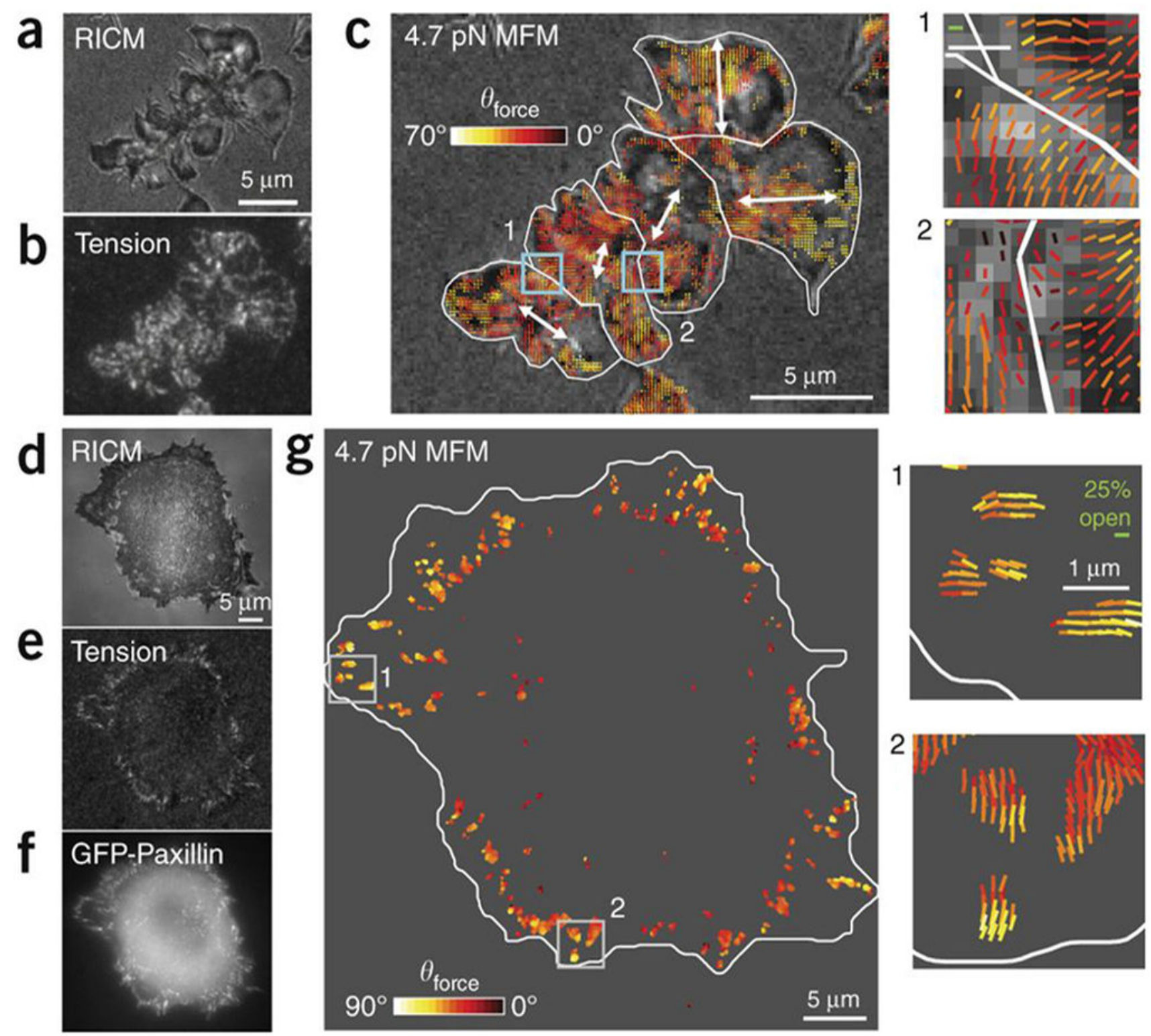

Figure 2: Molecular Force Microscopy is a general approach for measuring receptor forces.

(a) RICM, (b) $4.7 \mathrm{pN}$ tension, and (c) MFM image of a platelet aggregate comprised of five cells. The MFM image was overlaid onto the RICM, and white dipoles indicate average force orientation of individual platelets within the aggregate. The average force axes (white arrows) appear disordered ( $\mathrm{z}=0.225, \mathrm{p}=0.813$, Rayleigh test for uniformity). ROI 1 and 2 (represented on full MFM map by cyan boxes) show zoom-ins at the boundaries between platelets in the aggregate (green scale bar $10 \%$ open; white scale bar $500 \mathrm{~nm}$ ). 6 platelet aggregate MFM maps from $n=3$ independent experiments. (d) RICM, (e) $4.7 \mathrm{pN}$ tension, (f) GFP-Paxillin, and (g) MFM map of fibroblast seeded on a tension probe substrate for 30 min. The grey background in the MFM image represents pixels below an intensity threshold 
where the signal-noise ratio $<5$. ROI 1 and 2 show zoom-in to highlight the organization of integrin forces with FAs. 37 fibroblast MFM maps from $n=3$ independent experiments. 